\title{
TraduXio Project: Latest Upgrades and Feedback
}

\author{
Philippe Lacour ${ }^{1}$, Aurélien Bénel ${ }^{2}$ \\ 1 Universidade de Brasilia, Brazil \\ 2 Université de Technologie de Troyes, France \\ *Corresponding author: traduxio@philippelacour.net
}

\begin{abstract}
TraduXio (https://traduxio.org) is a digital environment for computer assisted multilingual translation that is web-based, free to use and based on open source code. Its originality is threefold: first, whereas traditional technologies are limited to two languages (source/target), TraduXio enables the comparison of different versions of the same text in various languages. Also, its concordancer provides relevant and multilingual suggestions through a classification of the source according to the history, genre and author. Finally, it uses collaborative devices (privilege management, forums, networks, history of modification, etc.) to promote a collective (and distributed) translation. TraduXio is designed to encourage the diversification of language learning and promote a reappraisal of translation as a professional skill. It can be used in many different ways, by a diversity of users. In this article, we will present the recent software update (its version 2.1) and illustrate how specific groups (language teaching, social sciences, literature) use it on a regular basis. In this paper we only briefly present the technology and its philosophy in order to concentrate more on the possible usecases of TraduXio, thus focusing on translators' feedback about their experience when working in this digital environment in a truly collaborative way.
\end{abstract}

\section{Keywords}

translation, computer-assisted, texts base, collaborative, multilingual, users' feedback, comparison 


\section{INTRODUCTION}

Digital Humanities can be generally defined as an endeavour to apply computer techniques to traditional Humanities and Social and Human Sciences, by bringing digital tools and methods, thus changing the way one builds, criticizes and spread knowledge [Gardiner \& Musto, 2015 ; Burdick et al., 2016 ; Berry \& Fagerjord, 2017 ; Shuster \& Dunn, 2020]. More than a trend, it implies a profound cultural revolution [Doueihi, 2011], with important normative aspects, including social and political issues - not to mention legal ones (open source, open access, open licences) [Le Deuff, 2017; Mounier, 2018]. It also constitutes an academic field of its own, geographically diversified, with linguistic specificities [Berra, 2020]. Now, one of the mains risks for its comprehension consists in mistaking technology and automation: indeed, the range of human-machine interaction is much richer than the mere delegation [Simondon, 1989, 2013]. For instance, not every technology of language is automatic: some only aim at (modestly) assisting humans in order to empower their intelligence of situations. One should therefore not yield uncritically to the current prestige of Artificial Intelligence, which reduces (by explicit principle or implicit postulate) the construction of meaning to a calculus of signification [Desclés, 2020], by trying to substitute human intelligence rather than simply help modelling it [Bénel, 2020].

As far as translation is concerned, Digital Humanities received both an early and critical reception [Cronin, 2013]. Translation Studies scholars can now analyze how these technologies are impacting the way translation is taught and theorized, and the way individuals actually translate texts. In this respect, one should not reduce translation technologies to translation memories and machine translation software [Olohan, 2017]. Indeed, machine translation has improved greatly over the past twenty years, thanks to more advanced statistical techniques, including the recent "neural" approaches at the heart of Artificial Intelligence, with a significant impact on research [Bowker \& Ciro, 2019]. Nonetheless, the postulates guiding this conception of technology remain questionable. Instead, translation can be considered a social practice relying on rigorous (justified) interpretation and collaboration, be it in the sphere of social networks [Desjardin, 2017] or for traditional translation of texts and discourse [Desjardin et al., 2020]. The project that we present here belongs to this alternative view of technology and semantics.

TraduXio (https://traduxio.org) was designed in 2006 by the Zanchin NGO (led by Philippe Lacour) and built in close collaboration with the Tech-Cico Lab of the University of Technology of Troyes, in France $^{1}$. Prof. Aurélien Bénel, the Head of Tech-Cico, has been developing open-source software focusing on computer assisted interpretation for years, particularly in the humanities and social sciences (https://hypertopic.org) $)^{2}$. TraduXio is a digital environment for computer assisted, collaborative and multilingual translation. It is a free device, with an open source code ${ }^{3}$. It is web based and not machine based which means it does not need to be downloaded. It was developed using innovative technology which is especially designed for multilingual translation and is particularly

\footnotetext{
${ }^{1} \underline{\text { https://recherche.utt.fr/technologies-for-cooperation-interaction-and-knowledge-in-collectives-tech-cico }}$

2 TraduXio benefited from both public (UNESCO, Organisation Internationale de la Francophonie, French Ministry of Culture (DGLFLF)) and private funding (IATIS NGO) for a total budget of approximately 60,000 euros (until now). This sum was mainly used to pay a computer scientist (first Diana Zambon, then Franck Eyraud) for coding. Other people participated, on a temporary basis, for web development (Assid Taounza), testing (Yuliya Goncharova) and fund raising (Any Freitas). A few graduate students from the Web Design class at the University of Technology of Troyes and from Telecom ParisTech also took part in the project.

${ }^{3}$ GNU/GPL3: technical details can be consulted online: https://github.com/Hypertopic/TraduXio
} 
suited to tackling the challenges of cultural (non-commercial, non-repetitive) texts. It promotes the creation of common goods guided by a logic of pooling (gradual feeding of the text base).

Drawing from the current state of the art, how can one situate TraduXio on the map of translation technologies? In fact, one can distinguish four logical possibilities, ranging from full automation ( $e$. g. Google Translate or DeepL) to human craftsmanship through two intermediary positions: human assisted machine translation (post-editing) and computer assisted human translation (CAT tools). There seems to exist some kind of continuity between the four options, in the sense that the human gesture can be taken separately, or captured, partially (a little vs a lot) or fully. In this perspective, machine translation would represent the natural goal of human translation (its implicit telos), the scientifically conceptualized and technically applied truth of this all too human operation. Although quite common, this interpretation mistakes the meaning of the difference between the two intermediary options, which is not a question of degree (more or less automation in the humanmachine mix) but of nature. Indeed, however thin it may seem, the line is very clear between two philosophies of technology: a conception that sees it as synonymous of automation, and another one that considers pure delegation of a human task as just a particular case (a poor one) of the rich variety of human-machine possible interactions (assistance, collaboration, etc.). In the case of translation, the line separates, on the one hand, projects like the former Google translation toolkit (in which human post-edition was instrumentalized to help improve statistical machine translation), and Matecat or OmegaT, which only offer machine translation as an option, while relying more on Translation Memories and human work.

But if the discrepancy in the philosophy of technology clearly appears, why is it that many people tend to overlook it and reduce it to a mere question of proportion - as if CAT tools were not yet as good as post-editing machine translation device? Perhaps one of the reasons lies in the fact that, however different, these visions of technology implicitly share the same principles concerning the nature of semantics. Namely, both consider linguistic meaning as mainly made of regularities - let alone exceptions. One should therefore enter the problem of translation by focusing on rules, which are to be applied to particular cases, like the chunk of text awaiting to be translated. In so doing, one frames the problem of meaning in a way that makes it open to measurement and therefore calculus (be it through the formalization of inference, as in grammatical machine translation, or of frequency of use, as in statistical machine translation). Although it benefits from more powerful algorithms, massive data sets (Big Data), or case-based empirical (deep) learning, contemporary Artificial Intelligence (be it symbolic or neural) is based on the same postulates that remain unquestioned, maybe because they are almost unconscious. What is at stake, in so doing, is the ambition to target singularities (this play, that poem), through the adequate application of rules. However, as far as complex, innovative cultural texts are concerned, technologies of translation come across enduring difficulties that are quite enlightening [Bénel et al., 2011].

If, on the contrary, one considers that singular variations are as much important as rules for languages, the question is whether technology is likely to be harnessed in order to help translate, e. g. the works of Shakespeare, Cervantès, Camus, etc. One would need to imagine not a data-based (as for translation memories) but a text-based technology (a concordance), meant for suggestion, assistance to interpretation, a collaborative environment strengthening human intelligence [Bénel et al., 2011]. For this very reason, TraduXio is not just another CAT tools. It rather belongs to the group of tools assisting human tasks, like contextual dictionaries, for instance (like Linguee), which do not pretend to replace human gesture and labour but to stimulate and empower it.

Indeed, as opposed to the vast majority of past and existing tools, TraduXio does absolutely not rely on the postulates of Natural Language Processing. For the latter, semantic units are sentences, translation consists of sending a message from a source to a target language (by pairs) and which focuses on universal rules - be they grammatical, statistical (including deep learning), or a 
combination of both. Instead, TraduXio considers that texts are the real semantic units, that translation consists in transforming a text into other texts in many different languages and that singularities are important for the technology of language (allowing only for contextualized generalization, and not universal rules). Consequently, TraduXio's uniqueness lies in its threefold originality.

(i) First, whereas traditional technologies are limited to two languages (source/target), TraduXio enables the comparison of different versions of the same text in various languages. The synoptic edition of multilingual texts is an old tradition with a very long history, pervading different cultures and tracing back (to our current knowledge) to the first translations of sacred texts (Bible, Qur'an, Buddhist holy scriptures) - figure 1.

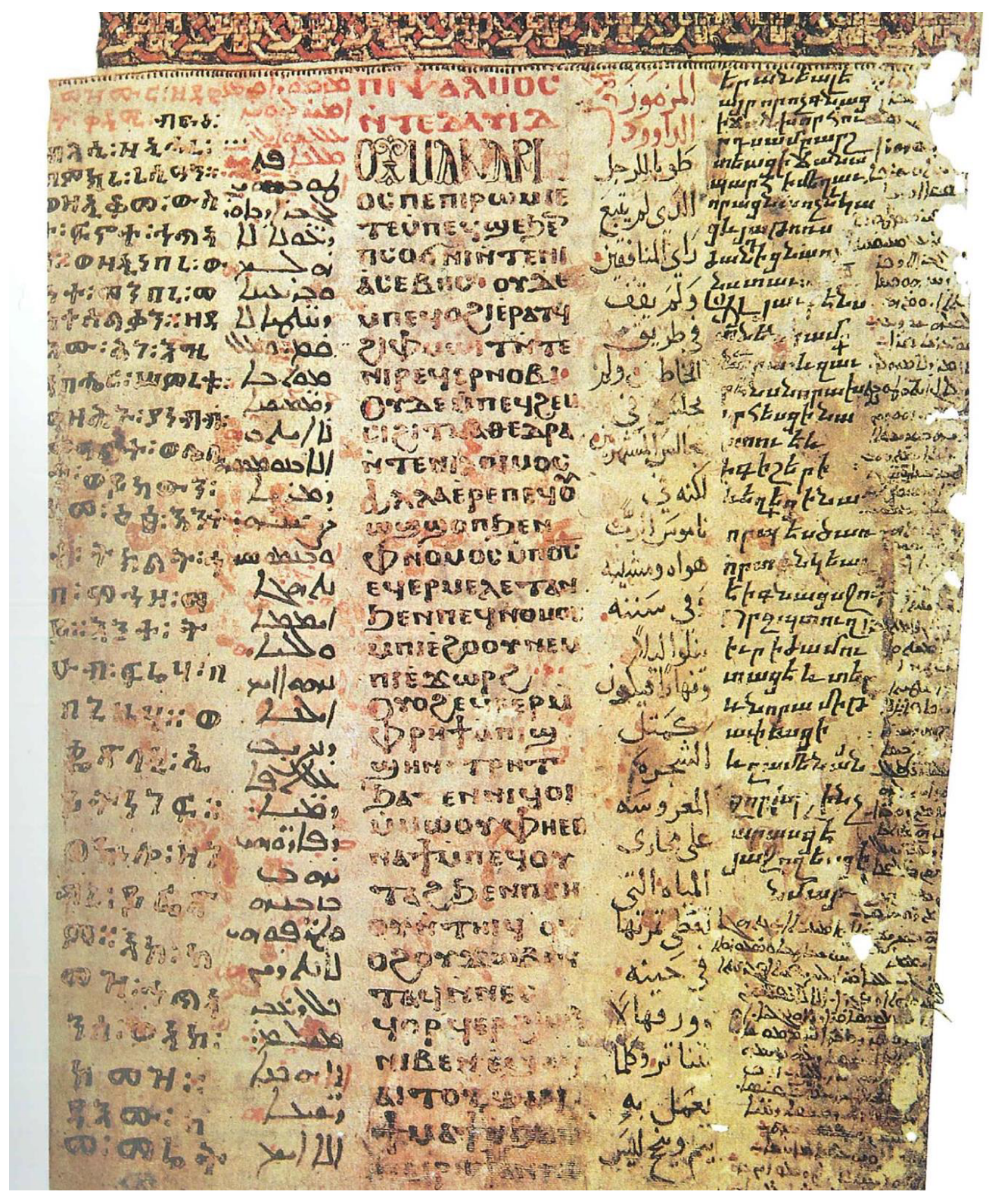

Figure 1: Manuscript of a Polyglot and polygraph Psalm collection. From left to right: Ge ez, Syriac, Coptic, Arabic, Armenian. Rome, Biblioteca Apostolica Vaticana, Barberini collection. 
TraduXio inspires itself from these first examples of texts alignments, which allow for systematic, translinguistic and transhistoric comparisons. In this respect, TraduXio is not inventing a technology but simply renovating an old traditional device by turning it digital (figure 2 ).

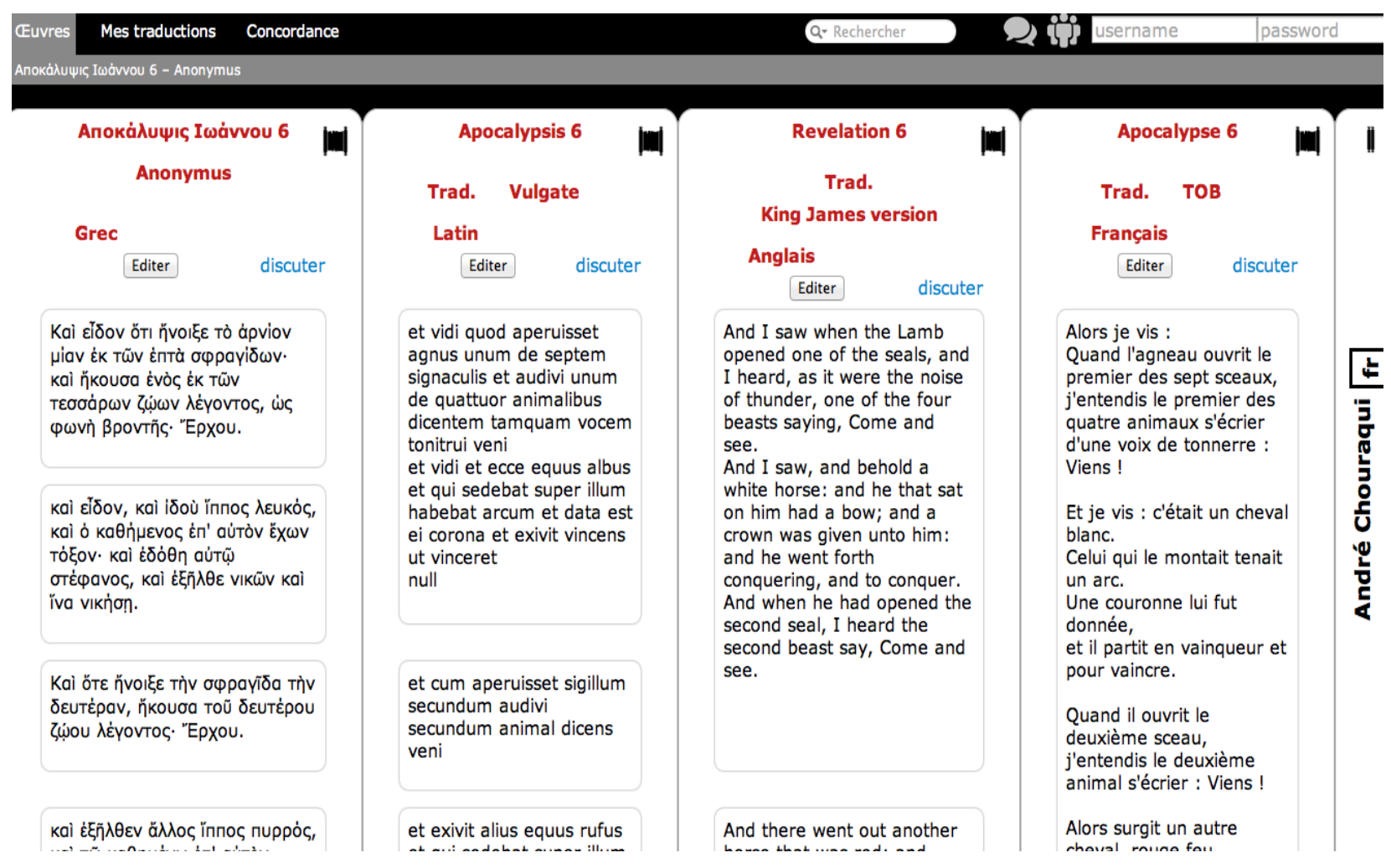

Figure 2. Different versions of St. John's Apocalypse; from left to right: Greek, Latin, English, French.

TraduXio is original because of its capacity for multilingual alignment of texts, whereas many software (e.g., YouAlign) limit themselves to bilingual possibilities (language pairs). But also because of its flexible segmentation. Indeed, since the original text is manually segmented, it allows for an adjustable division (much more open than Omega-T). For instance, in the above-mentioned translation (fig 2), none of the segments of the different columns are equivalent. The segments are not limited to a rigid format set by default (most of the time at the sentence level) and for the whole text: in a sonnet, it makes sense to split the stanzas differently, depending whether they are tercets or quatrains, for instance. This flexibility comes from the fact that, strictly speaking, TraduXio uses a text base and not a database [Bénel et al., 2011], the original text being considered from different standpoint —although TraduXio does not use the Hypertopic protocol (https://hypertopic.org), it works according to the same principle (the diverse perspectives on the same object corresponding to the various possible translations (in different languages) of the same original text).

(ii) Also, TraduXio's concordancer provides relevant multilingual suggestions through a classification of the source according to the history, genre, author, etc. Obviously, the size of the retrieved pieces of texts directly depend on the manual segmentation chosen (figure 3 ). 


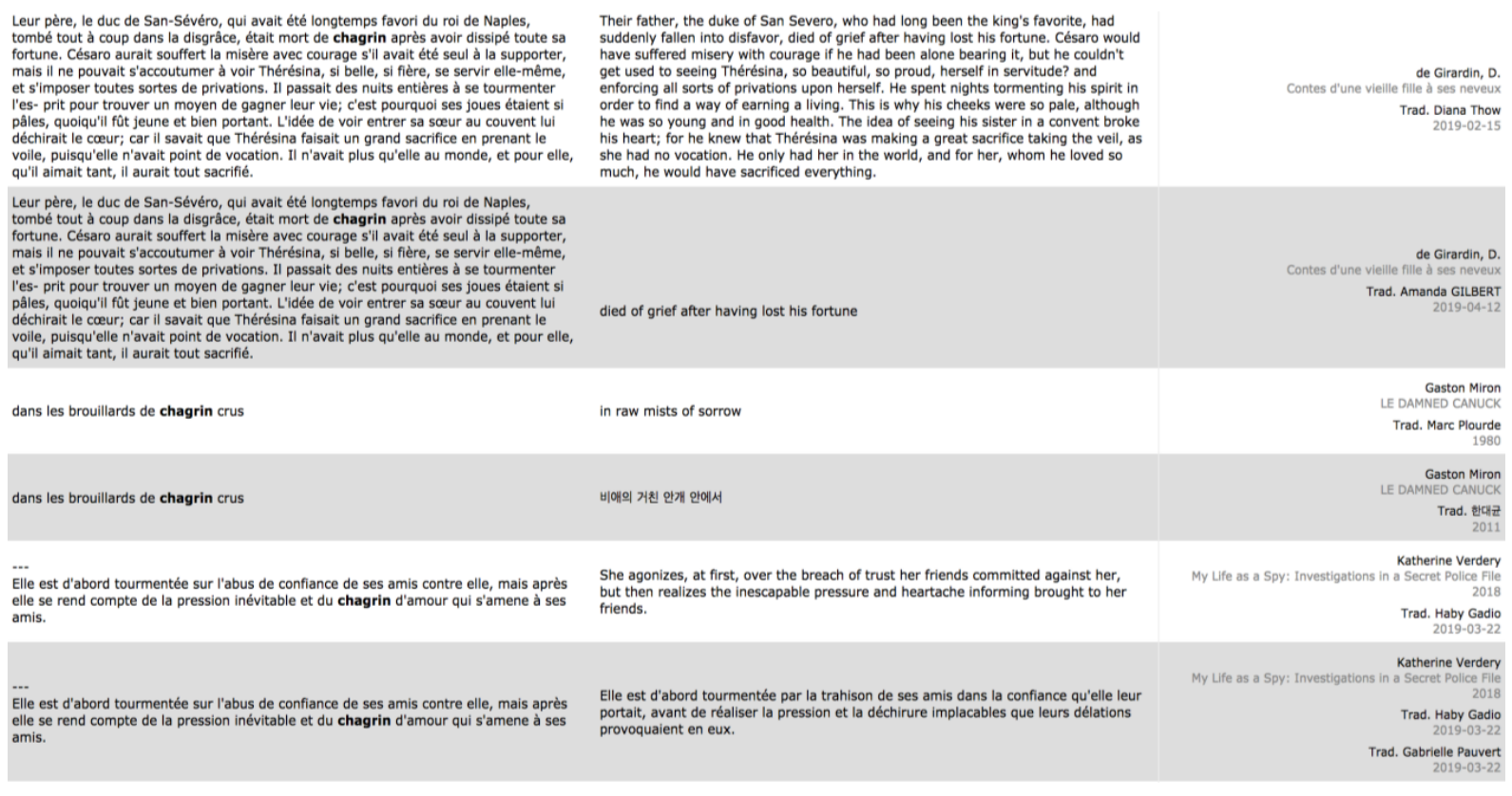

Figure 3. TraduXio's multilingual concordancer offers relevant suggestions

(iii) Finally, inspired by the strong collaborative spirit of "Web 2.0", it uses collaborative devices (privilege management, forums, networks, history of modification, etc.) to promote collective (and distributed) translation - for details, see [Lacour et al., 2010]. As it will be explained below, experience showed that some of these functionalities (chat, forum) were not satisfying enough and were temporarily left aside.

TraduXio's first draft version was designed in 2007 and a version 1.0 created in 2009 using technologies such as PHP and PostgreSQL with some basic functionalities. An improved version was ready in 2011 and included more efficient code and advanced collaborative functionalities (including tags, chats and forum). Unfortunately, its performance remained somewhat questionable. In 2014, the use of CouchDB allowed for a more robust and rapid version (2.0), which immediately gave more satisfaction to the users. Collaborative dimensions were added in the version 2.1 (in 2017), like privilege management, licensing, glossaries, localization (English, French, Spanish, Portuguese), although others have yet to be implemented (forum, history of modification).

From a technical standpoint, data are retrieved using a document-oriented data base processed by MapReduce (in particular, in order not to depend on elementary language treatment algorithms, which are limited to major languages). As far their copyright is concerned, one must distinguish three aspects. The code itself, being open source (GNU GPL3) is available online (on Github) at any time TraduXio being primarily a digital workbench and not a publishing device, the texts themselves are private by default, and therefore only visible to the main author (or to other users that are granted access to the document, through advanced privilege management). Other users, working on other translation projects, cannot access the original text nor the translation itself, although they can see chunks of it through the concordance (and legally, thanks to the quotation right). If one however decides to use TraduXio in order to publish one's translation, the opportunity is in that case given to choose and tag one's work with a license of one's choice (from full copyright to public domain through CC-type open licenses).

TraduXio is designed to encourage the diversification of language learning [Lacour et al, 2013] and to promote a reappraisal of translation as a professional skill. It can be used in many different ways 
by very diverse kinds of people. In this paper, we shall focus less on the technology and the legal aspects [Lacour and Freitas, 2011] than on the possible uses of TraduXio, thus focusing on translators' feedback about their experience when working in this digital environment in a truly collaborative way. We will successively study TraduXio's impact in the respective fields of language teaching, the humanities and social sciences, corpus building (and browsing), massive abstract translation and literature - each time trying to evaluate the current uses in order to suggest improvements and future orientations.

\section{LANGUAGE AND TRANSLATION TEACHING}

First of all, language students can use the platform to propose multilingual translations of assigned texts while language teachers can easily supervise the translation, propose corrections, compare drafts and evaluate students' questions and hesitations using the online interface. We shall now discuss how a translation teacher in Korea deals with TraduXio both inside and outside the classroom.

The teacher has succeeded in having students translate classical texts from French literature (Gaston Miron, Jacques Godbout, Eric-Emmanuel Schmitt, Gabrielle Roy) into Korean and English. He can also translate from Korean intro French

He can analyze four to six versions of the same translation on the same page, thus comparing the various efforts, focusing on the main mistakes and clever solutions. He can thus allow for hesitations and alternatives within a certain limit while at the same time ruling out mistaken interpretations.

The instructor can focus on the Korean language or draw the comparison across languages, by referring to the English version, thus evaluating the signification in a more dynamic and contrasted way (figure 4).

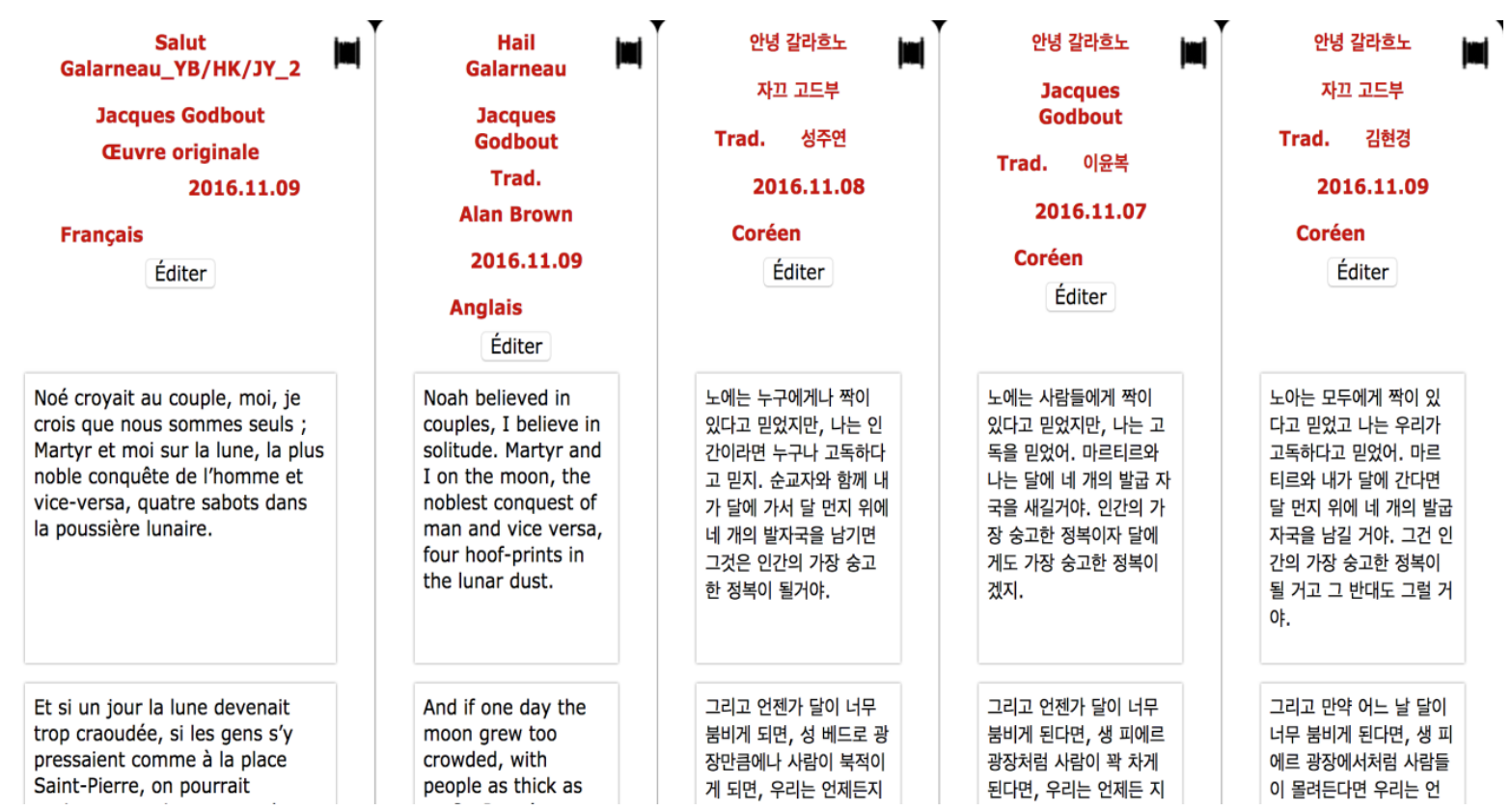

Figure 4. Translation of Jacques Godbout's text into Korean, English or in other Asian languages

The teacher also encourages his students to comparing their own work by themselves in a spirit of emulation, while suggesting critical remarks by way of questions, during the session in the classroom. By opening different windows, the language teacher therefore uses TraduXio's multilingual device to 
its fullest potential. This practice could lead to the future implementation of annotation (and/or underlining), and to the development of a new device for synchronous communication, in case of purely online translation.

Another interesting functionality consists in coordinating the translation by fixing a certain frame form the most prominent words, so as to enforce a certain convergence within a distributed translation process. Such is the role of the glossary, which can be constituted step by step in a collaborative and multilingual way, for a specific text (figure 5). This device could typically be further developed in order to expand a particular glossary to a wider corpus of texts, if, for instance, the same team of translators want to work on texts of the same author.

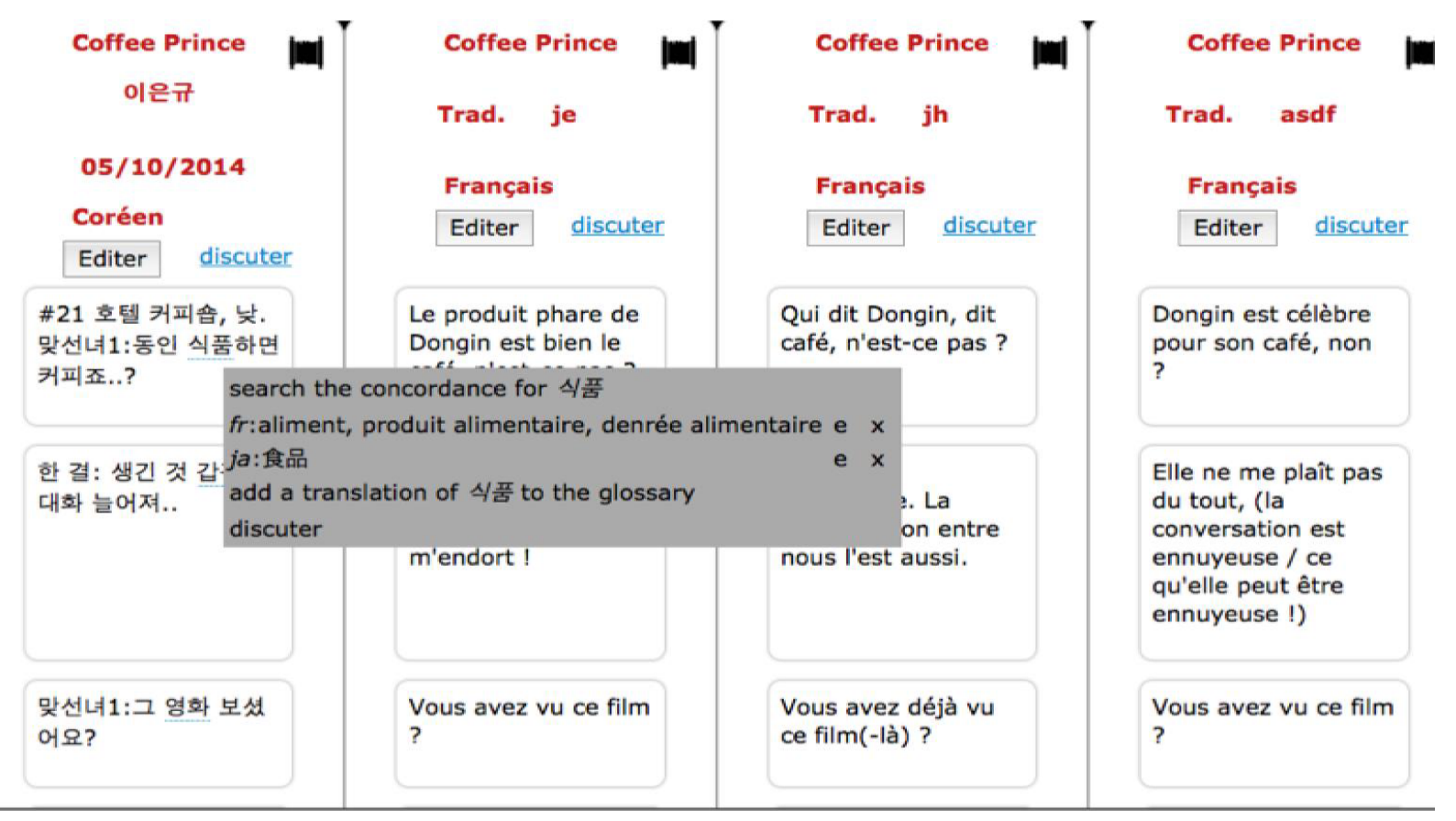

Figure 5. Do It (Yourself) With Others' Glossary.

Another teacher started using TraduXio for translation training because of the impossibility to meet physically in the classroom, for confinement reasons [Elbaz, 2021]. Before, she did not use any CAT tool, referring sometimes to machine translation as an example and a way to incite students to postediting activities. She chose TraduXio for its multilingual and collaborative dimension, working with a group of both French and Chinese students. She mentioned how she dealt with the communication issue, by harnessing a videoconference (through Microsoft Teams) with the students, beside the work on TraduXio - which required two screens. She divided her course in two sessions: the first one in little groups, discussing, translating and revising, and the second one with the entire class, to gather the different contributions and finish the translation. The first session was all the more important since the students had a different cultural background and translating practice, the Chinese focusing traditionally more on the source text and the French on the target text. And the teacher could follow the group sessions through the translation progress on the TraduXio website. The experience was quite satisfying, so that the teacher's next challenge is to translate a full literary novel with her next class (see below: V- Organizing Literature Translation Online). 


\section{TRANSLATING WITHIN THE HUMANITIES AND SOCIAL SCIENCES}

Second, TraduXio is also suited to use by scholars and Academic Departments particularly in the fields of Literature and the Social Sciences. Specialists can translate in a precise and critical way, respecting the highest academic standards, by creating multilingual glossaries or building a customized corpus thus creating a dedicated 'translation memory' for any topic or author. Users can manage text privileges, thus deciding which translations will be available to the public or remain private (and to what extent) and whether they can be modified or even cancelled. Leaders, who behave more like community managers than teachers, can thus distribute responsibility while avoiding miscarriages of translation due to inappropriate collaboration.

In this case, we shall refer to P. Lacour's experience as a philosophy teacher at the Federal University of Brasilia, where he has been leading a workshop for the translation of French philosophical classics into Portuguese. Here is an example of text of Bergson (figure 6), published for the first time in Portuguese (Lacour et al. 2020)

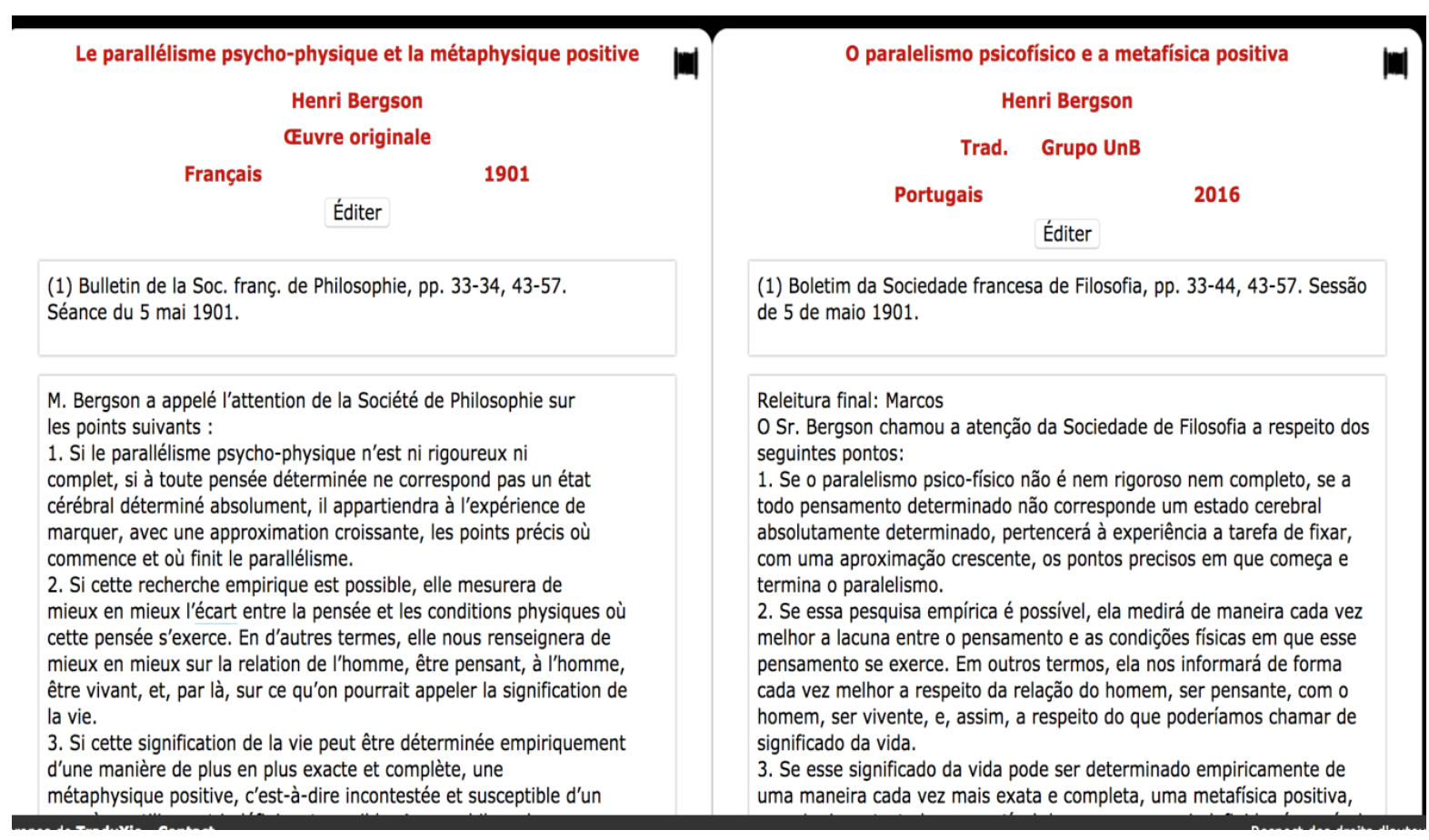

Figure 6. Translating Bergson into Portuguese

The group is composed of different kinds of students (translation studies, philosophy, even other disciplines like International Relations) who are both Brazilian and French-speaking (from Benin). None of the participants has full mastery of the three main required skills (French language, Portuguese language, philosophy). Therefore, everyone needs to rely on each other's capacities and engage in true collaboration through constructive dialogue.

The epistemic aspect of the discussions must be underlined, particularly how the group overcame disagreements through a process of progressive "fine tuning" in order to come up with a satisfying solution. Usually, every participant had to translate a part of the text, and to revise somebody else's translation, at home; the classroom session was dedicated to revision and collective decision making over hard cases. These difficulties implied a certain negotiation, the whole group having to consider the arguments, weigh the pros and cons, and eventually decide, sometimes through a democratic vote. The final version was however revised by a Professor who was at the same time Brazilian, a specialist of Bergson and a francophone. 
For the moment, there exist no device to import the source text or export the target text on TraduXio (one has to proceed through copy paste). But it would actually be very helpful to later publish the translation, as was done for the unpublished text of Bergson [Lacour et al., 2020].

\section{BUILDING A TRANSLATION CORPUS}

Thirdly, we will also insist on the experimentation carried out with the Faculty of Literature and Translation in Porto (Portugal).

The budget is really modest and the operation is restricted to opening a special digital text base inside the main TraduXio website, with a special access restricted to the members of the Centre for English Translation and Anglo-Portuguese Studies (figure 7).

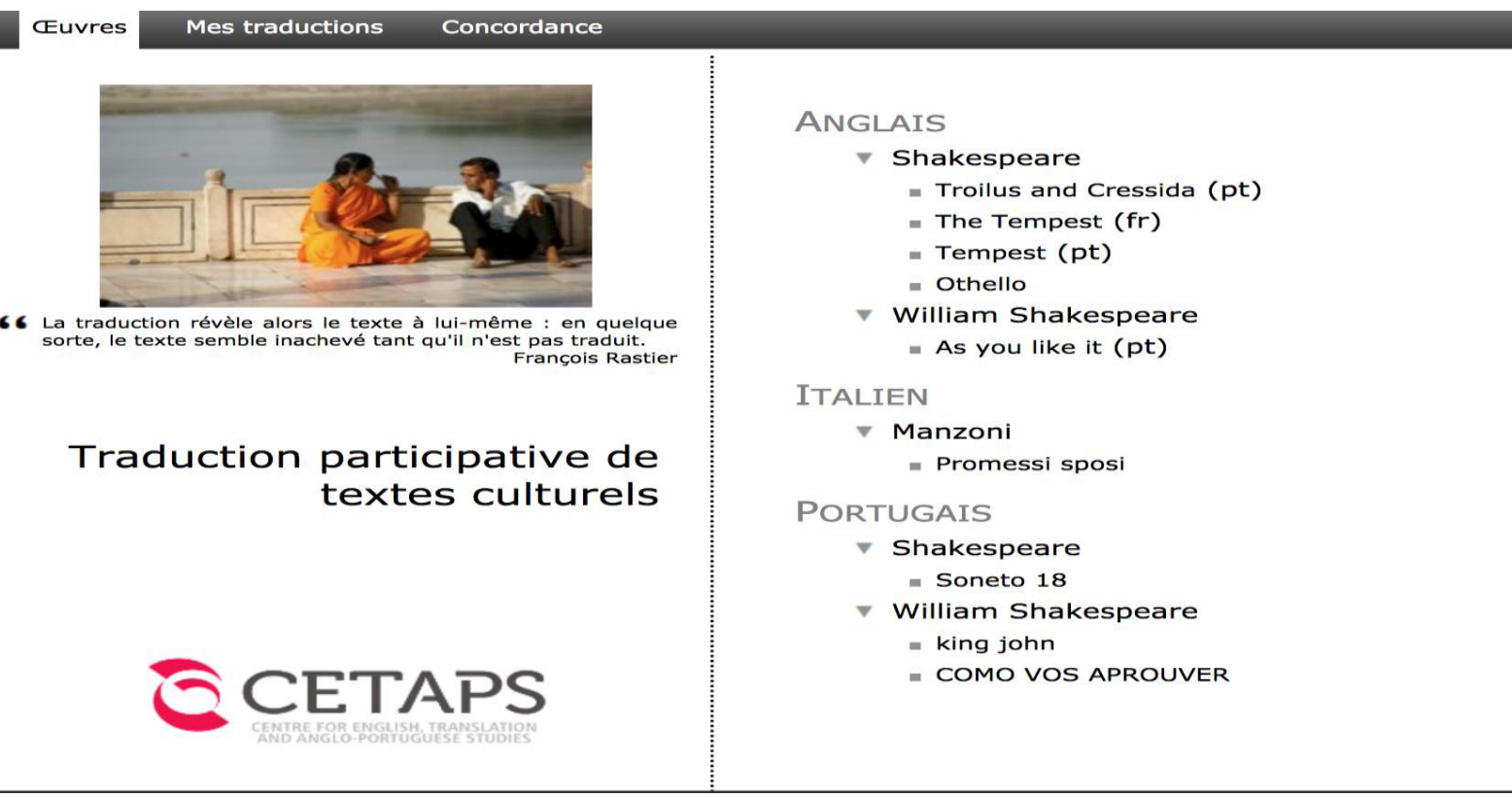

Figure 7. CETAPS' web page

The project consists in constituting a text base of (half of) the Portuguese translation of Shakespeare's plays. In so doing, the idea was to allow students to browse and look for concordances and also to foster corpus linguistics on Elizabethan theatre.

So far, the project only concerns a bilingual corpus, which all the members of the research group (and beyond) can fully consult in order to browse suggestive results. However, in the near future, it would be perfectly possible to enlarge the experiment to a multilingual corpus (inserting for instance the Spanish version of the plays) thanks to TraduXio's unique flexibility. Indeed, since TraduXio relies on a texts base rather than on a data base, one can simply add as many additional translations of the original text, in as many languages as one desires, thus improving the multilingual dimension of the corpus. Adding another translation language is an operation that can easily be repeated over time (by inserting a supplementary column), whatever the segmentation initially chosen, be it for the source text or for any of the translations. This is a genuinely subtle characteristic that data sets cannot offer. It could also be interesting for students and teachers alike to work together in the TraduXio environment on the translation of the rest of Shakespeare's plays which have yet to be retranslated. They could then constitute a reflexive and thorough glossary of the lexicon and make full use of the concordancer. This would be even more the case because they would use a multilingual corpus for inspiration. 


\title{
IV MANAGING THE TRANSLATION OF ABSTRACTS FOR AN INTERNATIONAL CONFERENCE
}

The fourth project deals more with pragmatic or technical translation than with their "literary" counterpart although there is a link between the two aspects. The International Association for Translation and Intercultural Studies asked TraduXio to help them prepare their international symposium in Hong-Kong in 2018, thanks to a specific web site (figure 8).

\section{TraduXio-IATIS}

\author{
Welcome to
}

\section{Collaborative \& multilingual translation tool for delivering an English-into-Chinese book of abstracts for the IATIS Hong Kong Conference (July 2018)}

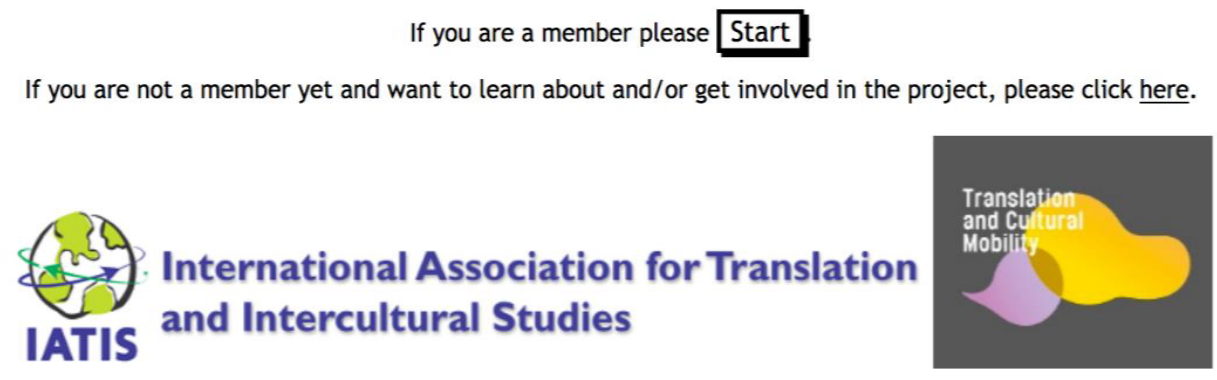

Figure 8. The TraduXio-IATIS customized digital workbench.

This was a very challenging project requiring more funding and manpower than the others. The operation is quite complex, since it involves taking abstracts and metadata from the Easychair platform then translating them and sending them back in preparation for them being located on the IATIS website. The TraduXio environment was chosen because of its unique capacity to deal with a multilingual workflow and to distribute translation. In this project three hundred original English versions had to be translated into Chinese. This required a team of about forty properly trained translators (which involves a tutorial, the right training and the localization of the software). A supervisor was needed to act as both trainer and project and community manager with technical code skills (figure 9). A set of extra-functionalities was also needed, including an automatic importing and exporting device for abstracts (from the IATIS symposium's 'comfortable' collaborative space to the customized IATIS-TraduXio web environment and back), a set of fixed "roles" or "tasks" (translation begun, translation completed, under revision, revision completed, finalizing (double check), finalization completed), automatic segmentation per sentence. 


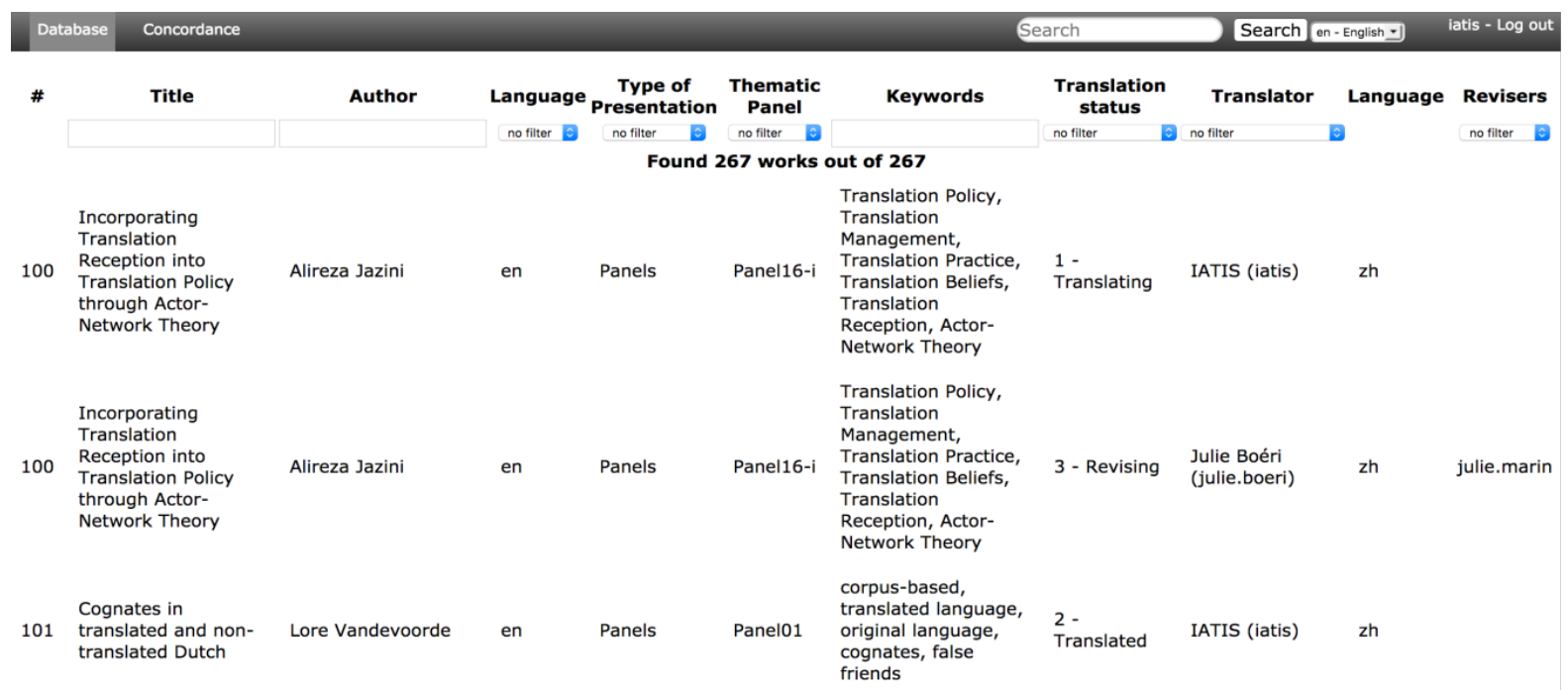

Figure 9. Role distribution among translators working on the IATIS-TraduXio's interface

The fundamental asset of the operation is for IATIS to begin shaping a multilingual texts base that can be gradually fed and reused for its future symposiums.

This experiment was however quite demanding for the TraduXio team, because it involved an important development of functionalities that bended the project towards automation (importing and exporting device, sentence segmentation and alignment, etc.). Such an orientation is obviously not akin to TraduXio's core philosophy, not to mention the fact that a lot of other software are well capable of such tasks. However, it also shows that it is possible to integrate TraduXio within a more automatic workflow and project.

\section{ORGANIZING LITERATURE TRANSLATION ONLINE}

The TraduXio digital environment can of course finally be used to translate literature as it was specifically designed for this and particularly for semantically difficult texts (religion, philosophy, etc.). Two kinds of experimentation have been led in this respect. The first project was led by a single translator, Yuliya Goncharova, interested in translating French literature into Russian [Lacour and Goncharova 2011]. It helped identify specific needs related to the length of the texts, their lexical richness and their original setting and page layout. The translator worked on Rimbaud's famous early poem, "Le dormeur du val" using TraduXio's concordancer for lexical and semantic analysis (figure 10). This tool is particularly helpful when it comes to metaphors and images, when a translator needs suggestions and can use the concordancer to retrieve relevant ideas taken from other literary sources. The advantage (for truncation, homonyms and polysemic terms) and limits (lack of recognition of vowel alternation which is very common in Russian; the inability to identify suppletion) are also clearly underlined.

C'est un trou de verdure où chante une rivière Accrochant follement aux herbes des haillons D'argent; où le soleil, de la montagne fière, Luit : c'est un petit val qui mousse de rayons.
В зеленом разломе, где речка поет, Росою сплетая траву в паутину, Где с гордой вершины солнце свет льет, Щедро купая в том свете долину.

Figure 10. Translation of the beginning of Rimbaud's "Le dormeur du val" into Russian 
The second project was led by Daniel Henkel and involved coordinating groups of translators (both graduate students and teachers) from three different academic institutions - University Paris 8 (France), University of Berkeley (USA) and University of Florence (Italy) - for six months plus one intensive week [Henkel and Lacour 2020]. This time TraduXio was mainly used for confronting ideas and critical discussion (also using other canals of communication) and involved many students discovering for the first time the advantages and limits of collective translation and the necessity of examining one's own point of view. The students were organized in small groups of 3-4 people to translate English short stories into French and Italian while often comparing their ideas with other groups before finally harmonizing their work under the supervision of the faculty members (figure 11).

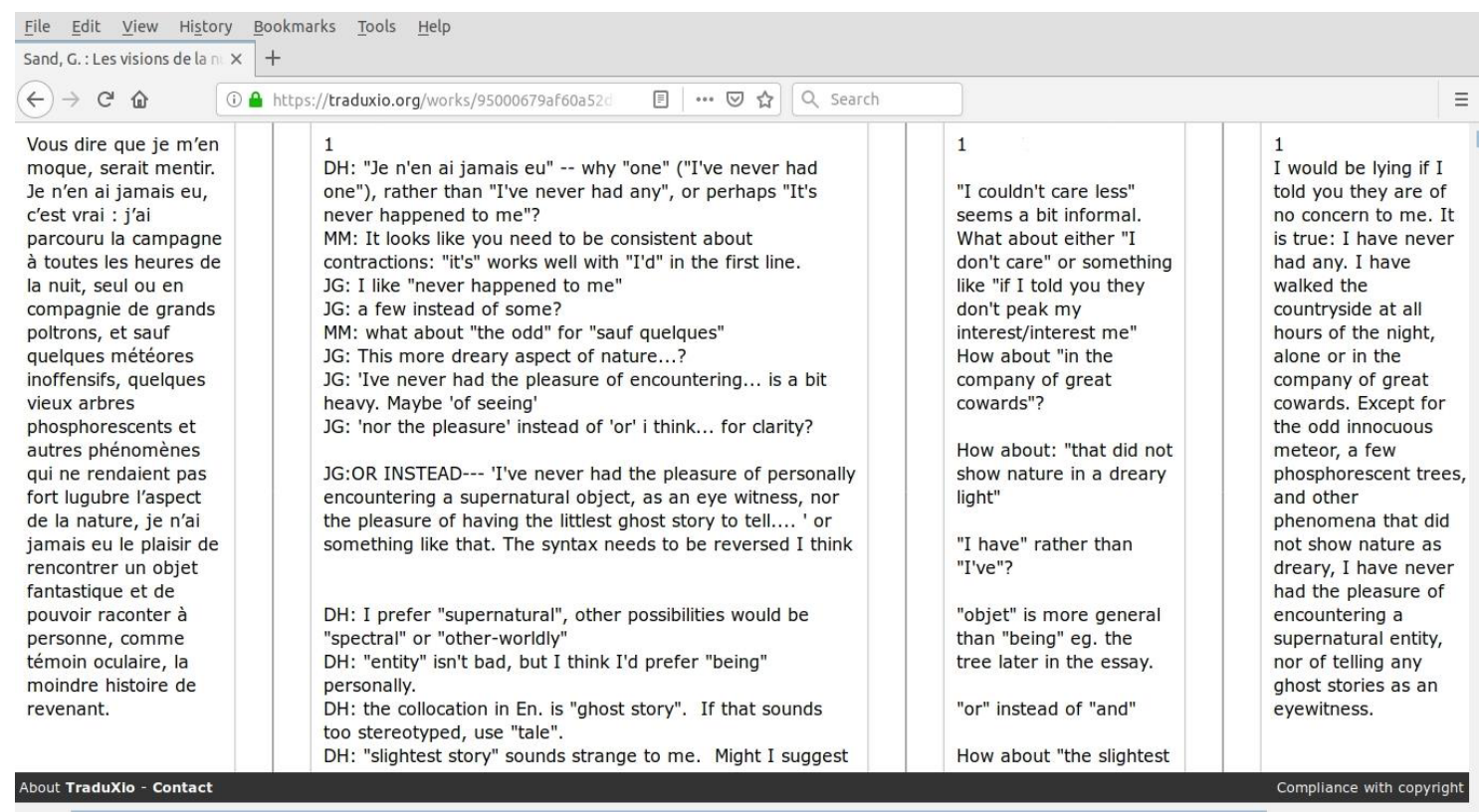

Figure 11. The faculty suggestion and discussion column

There were numerous inevitable problems. These included a lack of a synchronous communication device (students used Skype or WhatsApp to discuss their work, sometimes even pasting it into a Google doc), the lack of a comment function, the fact that it is impossible to track changes (history of modifications) and edit the text (with bold, italics, etc.) and the scale of project allied to the number of participants. However, a vast majority of participants agreed that multilingual collaborative online translation had been a positive and enjoyable experience which was also mutually enriching though the construction and confrontation of diverse points of view on the same object.

\section{CONCLUSION}

To sum up, those examples clearly show the maturity of the TraduXio digital environment, which is both rapid and robust in its current 2.1 version. Each case gives an illuminating insight of its possible applications, while highlighting its advantages (it is multilingual, collaborative and suggestionoriented) and limits (synchronous communication, the lack of a more advanced collaborative device).

TraduXio's main asset consists in the importance given to the texts. Indeed, if one accepts the idea that the texts are the real semantic units [Rastier, 2001], one imagines a very different technology of translation, based on suggestion, comparison and interpretation. The multilingual digital environment 
helps empower groups of translators rather than reduce their input to post-editing « cleaning » tasks. Far from being synonymous of a "waste of time », the assistance, if the community is properly managed, might lead to substantial speed, especially when precise translation is needed, like it is the case for cultural texts (with difficult semantics) - indeed, during various presentations, the software also raised the attention of specialists of « technical» (e.g., legal) translation.

The project also faces various challenges, from a methodological, technological and collaborative point of view. In particular, because the TraduXio team worked with a shoe-string budget, elbowing its way through the crowd of Web 2.0 applications and Web 3.0 fads - not to mention the IA contemporary wave - it chose to focus on the heart of its originality (the multilingual concordancer and texts base). It therefore left aside many aspects that would help improve the general ergonomy: device to import, export, edit the texts; annotation functionalities, synchronous (chat, audio- or videoconference) and asynchronous communication aspects (forums), etc.

Users' feedback is obviously crucial to gather information in order to imagine future developments of the TraduXio project. Students (of the Web design class) from the University of Technology of Troyes (France) also contributed to this study by participating in defining the next steps for the process, thus paving the way for future improvements (https://github.com/Hypertopic/TraduXio/issues). In particular, two functionalities are of great importance in the near future, and both rely on the same piece of technology. The first is more oriented towards erudition as it allows for reference to be made to the original version of the source text, for instance, Kant's Critique of Pure Reason: A (first) or B (second edition). It could become of immense value for philological use of TraduXio (figure 12).

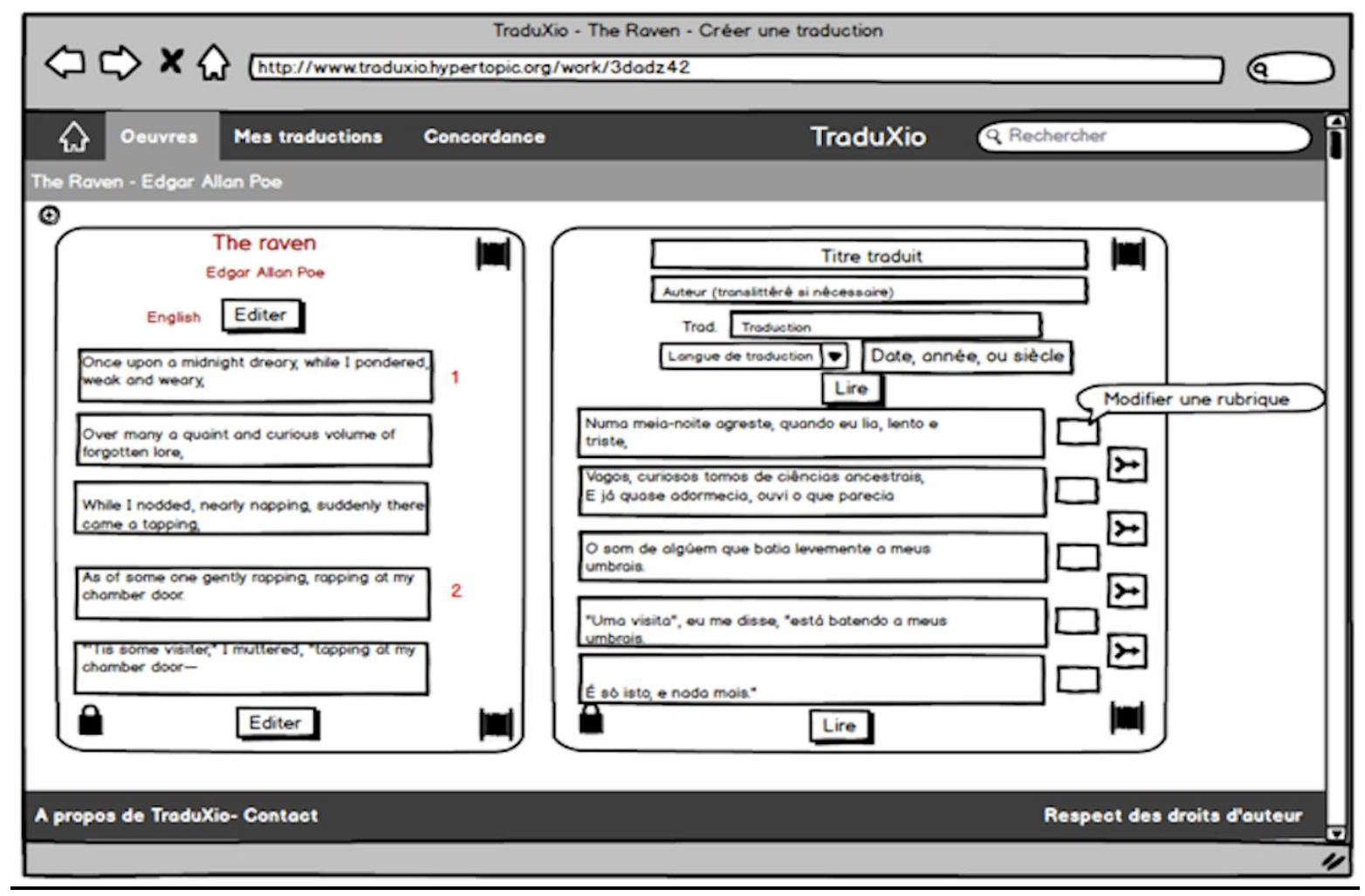

Figure 12. TraduXio' possible reference to the original version of Poe's poem "The Raven" 
Now, as often happens in computer science, one functionality can lead to very different uses and user adoption (fork). The same idea of referring to the original version of a document is relevant to mention the exact chronological location of a specific subtitle in a video. TraduXio could therefore also be used - this time in an industrial way (culture, entertainment) - for digital multilingual subtitling (figure 13).

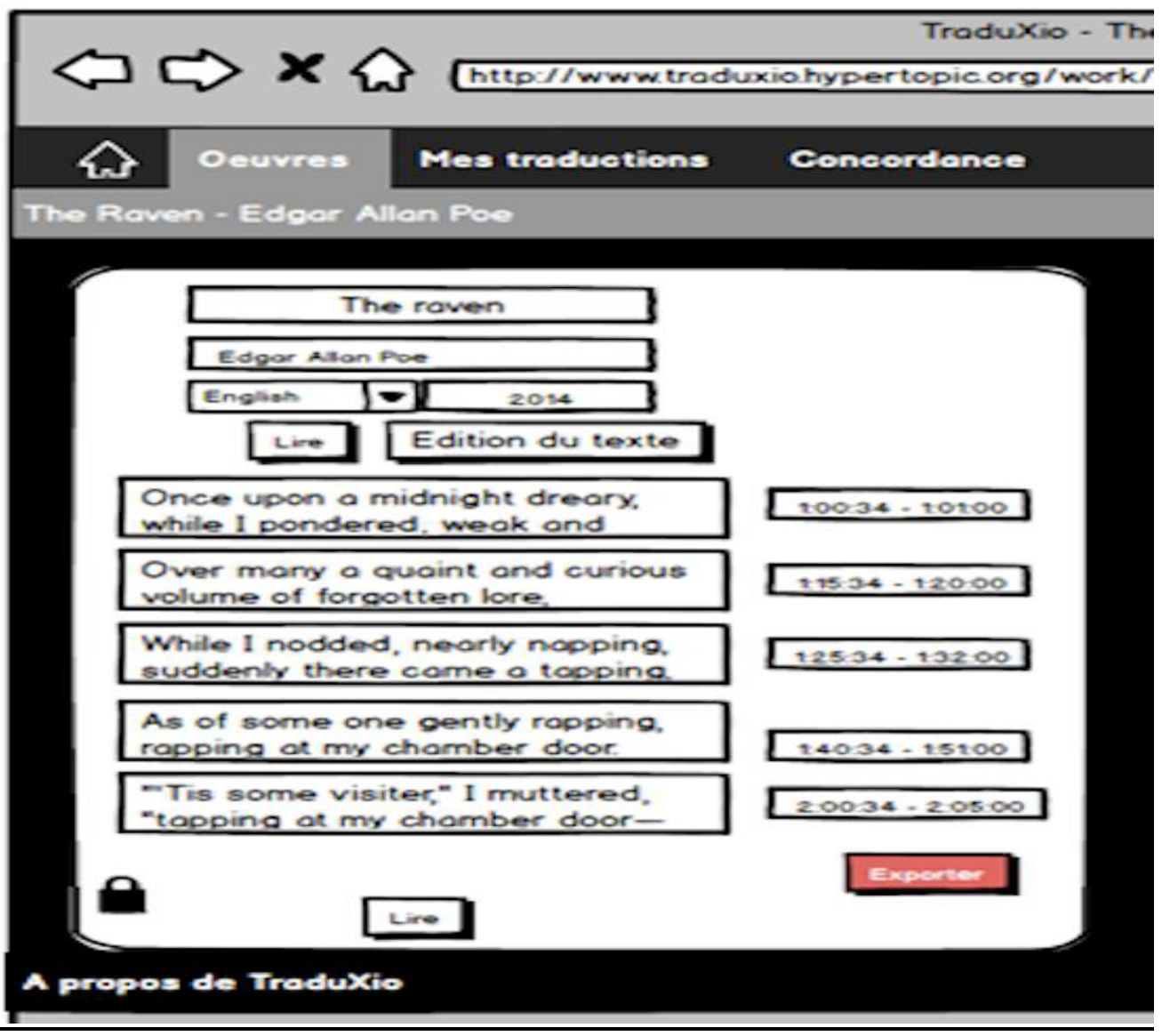

Figure 13. TraduXio's possible use for the translation of video subtitles

\section{References}

Bénel A. Modéliser ce qui résiste à la modélisation. Revue ouverte d'intelligence artificielle. 2020;1 (1):71-88.

Bénel A., Lacour P. and Eyraud F. Towards a Collaborative Platform for Cultural Texts Translators. Pierre Maret (ed). Virtual Community Building and the Information Society: Current and Future Directions. IGI Global (Hershey, Pennsylvania), 2011.

Berra A. Éditorial. Donner à lire les humanités numériques francophones (1) et (2). Humanités numériques. 2020;1-2.

http1 https://journals.openedition.org/revuehn/508

http2 https://journals.openedition.org/revuehn/507

Berry D.M. and Fagerjord A. Digital Humanities: Knowledge and Critique in a Digital Age. Polity (Cambridge), 2017.

Bowker L. and Ciro J. Machine translation and global research: towards improved machine translation literacy in the scholarly community. Emerald Publishing Limited (United Kingdom), 2019.

Burdick A., Drucker J., Lunenfeld P., Presner T. and Shnapp J. Digital Humanities. MIT Press (Cambridge), 2016.

Cronin M. Translation in the Digital Age. Routledge (New York), 2013.

Desclés J.-P. Vers un calcul des significations dans l'analyse des langues. Revista de Filosofia Moderna e Contemporânea. 2020;8 (1). https://periodicos.unb.br/index.php/fmc/article/view/31016.

Desjardins R. Translation and Social Media: In Theory, in Training and in Professional practice. Palgrave Macmillan (London), 2017. Desjardin R., Larsonneur C. and Lacour P. When Translation Goes Digital. Case Studies and Critical Reflections. Palgrave (London/New York), 2020. 
Doueihi M. La grande conversion numérique. Seuil (Paris), 2008 (Digital cultures ([American ed.].). Cambridge, Mass: Harvard University Press, 2011).

Elbaz P. Interactivité, co-construction : quand l'outil numérique dé-hiérarchise le rapport entre enseignant.es et étudiant.es en langue. Ouvrard L. and Szende T. (eds). Distances apprivoisées: l'enseignement confiné des langues étrangères. Éditions des Archives Contemporaines (Paris), 2021 (forthcoming).

Gardiner E. and Musto R. The Digital Humanities. Cambridge University Press (Cambridge, UK), 2015.

Henkel D. and Lacour P. Collaboration Strategies in Multilingual Online Literary Translation: the Case of the TraduXio Environment. Desjardin R., Larsonneur C., Lacour P. (eds.), When Translation Goes Digital. Case Studies and Critical Reflections. Palgrave (London/New York), 2020.

Lacour P., Bénel A., Eyraud F., Freitas A. and Zambon D. TIC, Collaboration et Traduction: vers de nouveaux laboratoires de translocalisation culturelle. Meta. 2010; 55(4):674-692. https://www.erudit.org/fr/revues/meta/2010-v55-n4-meta4003/045685ar/.

Lacour P., Freitas A. Translation and the New Digital Commons. Métiers et technologies de la traduction: quelles convergences pour l'avenir ? Proceedings of the Tralogy symposium. March 3-4 2011 (Paris). http://lodel.irevues.inist.fr/tralogy/index.php?id=150.

Lacour P. and Goncharova Y. Traduxio: nouvelle expérience en traduction littéraire. Traduire (Société Française des Traducteurs). 2011; 225. https://journals.openedition.org/traduire/94.

Lacour P., Bénel A., Eyraud F., Freitas A. and Zambon D. Enhancing Linguistic Diversity through Collaborative Translation. TraduXio: an Open Source Platform for Multilingual Workflow Management in Media. Elin Haf Gruffydd Jones and Enrique Uribe-Jongloed (eds). Social Media and Minority Languages. Convergence and the Creative Industries. Multilingual Matters (Bristol, Buffalo, Toronto), 2013.

Lacour P., Oliveira Chaia J., . Randal e Zitti S. C., Mucury Teixeira M., Alves Teixeira M., Ribeira Braatz A., Elisa Malby Carvalho Augusto E. And Morato Pinto D. C. "O Paralelismo Psicofísico e a Metafísica Positiva", de Henri Bergson. Revista de Filosofia Moderna e Contemporânea. 2020;8(1):457-484. https://periodicos.unb.br/index.php/fmc/article/view/30414.

Le Deuff O. Les humanités digitales : historique et développements. Iste éditions (London), 2017.

Mounier P. Les humanités numériques : Une histoire critique. Éditions de la Maison des sciences de l'homme (Paris), 2018.

Olohan M. Technology, translation. Baker, M. \& Saldana, G. (Eds). Routledge Encyclopedia of Translation Studies ( $3^{\text {rd }}$ ed) Routledge (New York), 2020: 574-579

Rastier F. Arts et sciences du texte. PUF (Paris), 2001.

Schuster K. and Dunn S. International Research Methods in Digital Humanities. Routledge (London), 2020.

Simondon G. Du mode d'existence des objets techniques. Aubier (Paris), 1989.

Simondon G. L Individuation à la lumière des notions de forme et d information. Charles Millon (Grenoble), 2013. 